\section{IMPLANTS VIA AN \\ INTUITIVE INTERFACE}

The Bien-Air Chiropro L ultraefficient, extremely versatile implantology system leaves nothing to chance.

The Chiropro L has been designed with efficiency and comfort in mind. The intuitive interface can be managed by the control pedal. The patented peristaltic pump with disposable irrigation lines and insertion support ensures easy handling and optimum infection control.

The Chiropro L System includes the MX-LED self-ventilating motor, which is the most powerful on the market to offer LED lighting at equal intensity at both high and low speeds. Incorporating seven of the leading brands of implants with their complete sequences the Chiropro L can also be customised to your individual requirements.

With the world's first internal irrigation system the 20:1 contraangle handpiece incorporates the smallest head on the market allowing access to the tightest of areas. Fitted with an exclusive double optical glass rod system, the handpiece provides uniform lighting of the operative field.

This user-friendly system has proved to meet the demands of implantologists worldwide and more.

Contact Bien-Air on 01293550 200 or visit www.bienair.com.

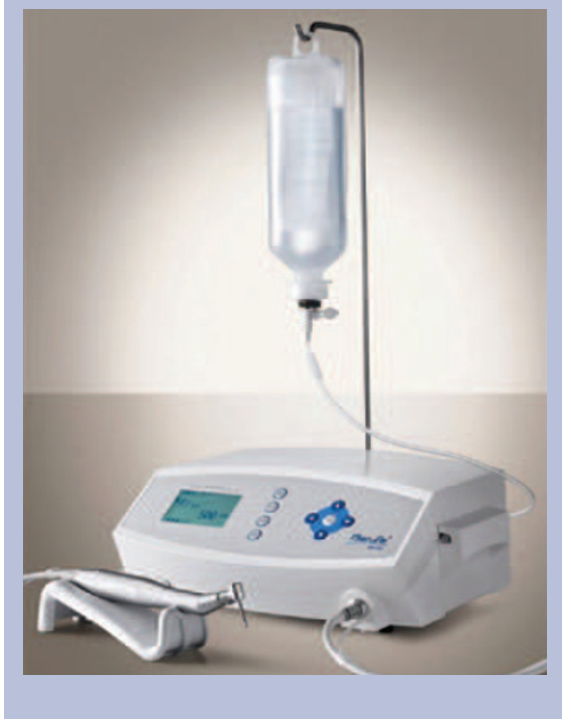

\title{
IMPLANTS WITH OUTSTANDING SAVINGS
}

Implant Complete from CosTech Elite is an all-inclusive dental restoration package that presents an outstanding saving for both clinicians and patients.

Implant Complete offers a comprehensive restoration service including a laboratory analogue and a titanium engagement platform for superior strength and durability. The custom CAD/CAM zirconia abutment and crown or bridge also offers a number of benefits, with an all-ceramic construction providing outstanding aesthetics and biocompatibility.

As the requirements of any patient are always very specific, CosTech Elite can include any combination of these components as necessary at a reasonable fixed cost. The Implant Complete service can also be further customised to incorporate a soft tissue model or location jig for easier, naturallooking integration with the patient's existing dentition.

With a fixed price reflecting a saving of around £100 per unit, as well as a worldwide guarantee on all restorations and a one week turnaround time courtesy of CosTech Elite's superior Elite 7 service, Implant Complete is an exceptional and affordable means of bringing your patients the perfect smile they deserve.

Call 01474320076 or email info@ costech.co.uk for more information.

\section{FOR ALL YOUR ORTHODONTIC NEEDS}

NSK offer an exceptional range of slow and high-speed instruments ideal for use in orthodontic procedures.

For effective cleaning and polishing before bond-up, during treatment when changing arch wires, and after de-bonding, NSK offer a range of ultrasonic scalers, air scalers and air polishing systems.

Prophy-Mate neo is a reliable and user-friendly air polishing system perfect for orthodontists, with quick and easy connection to all major couplings. When combined with FLASH pearl, NSK's effective prophy cleaning powder, Prophy-Mate neo quickly and safely cleans and polishes to deliver sparkling results.

The Varios 970 ultrasonic scaler is a stylish, compact unit with an extensive range of over 70 tips to meet most clinical needs. The newly developed iPiezo engine provides stable vibration and power with excellent illumination of the treatment area guaranteed by twin LEDs. The Varios 570, or the Varios 370 , are more compact units specifically designed for portability between dental units. Additionally, the Varios 170 is available as a built-in unit, where all its functions can be controlled via NSK's new MultiPad. For those looking to air scale, NSK's new Ti-Max S970 offers an adjustable power range, delivering effective and powerful scaling in a solid titanium body, providing a steady, comfortable grip.

Contact NSK on 08006341909 or your preferred dental supplier. www.nsk-uk.com.

\section{BEAT THE STAMP PRICE HIKE WITH A FRANKING MACHINE}

After record stamp price hikes by Royal Mail, dental practices could save hundreds of pounds a year by using a SMART franking machine.

Using a SMART franking machine enables dental practices to access all Royal Mail's services, including recorded delivery, special delivery, packet post and parcels, at a discounted rate, and could ensure that practices are not unduly affected by the increase in stamp costs.
Royal Mail has kept the prices for their franked mail competitively low in a bid to encourage business customers to pre-pay for their postage. The franking price for a first class letter is $44 \mathrm{p}$ whereas a first class stamp now costs 60p.

There are also time savings and an added professional touch from an advertising logo and return address on each envelope sent out.

www.themailingroom.com 